Article

\title{
PDMS-Based Microdevices for the Capture of MicroRNA Biomarkers
}

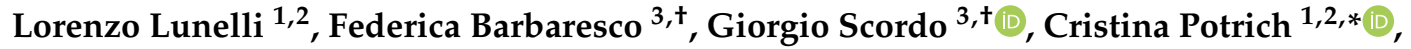 \\ Lia Vanzetti ${ }^{1}$, Simone Luigi Marasso ${ }^{3,4}\left(\mathbb{D}\right.$, Matteo Cocuzza ${ }^{3,4}{ }^{(\mathbb{D}}$, Candido Fabrizio Pirri ${ }^{3,5}$ \\ and Cecilia Pederzolli ${ }^{1}$ \\ 1 Fondazione Bruno Kessler, Center for Materials and Microsystems, via Sommarive 18, \\ I-38123 Povo (Trento), Italy; lunelli@fbk.eu (L.L.); vanzetti@fbk.eu (L.V.); pederzo@fbk.eu (C.P.) \\ 2 CNR_Consiglio Nazionale delle Ricerche, Istituto di Biofisica, via alla Cascata 56/C, \\ I-38123 Povo (Trento), Italy \\ 3 Department of Applied Science and Technology, Politecnico di Torino, Corso Duca degli Abruzzi 24, \\ 10129 Torino, Italy; federica.barbaresco@polito.it (F.B.); giorgio.scordo@polito.it (G.S.); \\ simone.marasso@polito.it (S.L.M.); matteo.cocuzza@infm.polito.it (M.C.); fabrizio.pirri@polito.it (C.F.P.) \\ 4 CNR-IMEM, Parco Area delle Scienze 37a, 43124 Parma, Italy \\ 5 Istituto Italiano di Tecnologia, Center for Sustainable Future Technologies @ PoliTo, Corso Trento 21, \\ 10129 Torino, Italy \\ * Correspondence: cpotrich@fbk.eu; Tel.: +39-0461-314-605 \\ + These authors contributed equally.
}

Received: 5 May 2020; Accepted: 30 May 2020; Published: 2 June 2020

\begin{abstract}
The isolation and analysis of circulating biomarkers, the main concern of liquid biopsy, could greatly benefit from microfluidics. Microfluidics has indeed the huge potentiality to bring liquid biopsy into the clinical practice. Here, two polydimethylsiloxane (PDMS)-based microdevices are presented as valid tools for capturing microRNAs biomarkers from clinically-relevant samples. After an extensive study of functionalized polydimethylsiloxane (PDMS) properties in adsorbing/eluting microRNAs, the best conditions were transferred to the microdevices, which were thoroughly characterized. The channels morphology and chemical composition were measured, and parameters for the automation of measures were setup. The best working conditions were then used with microdevices, which were proven to capture microRNAs on all channel surfaces. Finally, microfluidic devices were successfully validated via real-time PCR for the detection of a pool of microRNAs related to non-small cell lung cancer, selected as proof-of-principle. The microfluidic approach described here will allow a step forward towards the realization of an efficient microdevice, possibly automated and integrated into a microfluidic lab-on-a-chip with high analytical potentialities.
\end{abstract}

Keywords: PDMS microdevice; surface functionalization; microRNAs

\section{Introduction}

In recent years, the concept of a non-invasive but specific diagnostic analysis, able to detect minimal amount of biomarkers at the early stages of a pathology, has grown enormously. In fact, the ability to tailor medical care to an individual patient is connected to the possibility of collecting information on that individual, starting from low amount of biological samples, to be collected throughout time. To this regard, liquid biopsy is gaining significant attention for biomarker analysis and discovery, especially for pathologies like cancer [1,2]. In this context, microfluidic technologies offer great potential to revolutionize the way for sampling, sample separation, mixing, chemical reaction, and detection of biomarkers in clinical settings. Several examples of microfluidics applied to biomedical field are present in the literature [3-5], from the identification of cancer biomarkers such as 
circulating tumor cells, circulating tumor DNA and extracellular vesicles [6,7] to single cell analysis [8] and organ-on-a-chip technology [7,9], to nucleic acid diagnostics [10-12].

Among different materials suitable for microsystem fabrication, polydimethylsiloxane (PDMS) has been widely used in a variety of microfluidic applications, including for manufacturing lab-on-a-chip and micro total analysis systems [13]. PDMS, a silicone-based organic elastomer, is indeed a low-cost, flexible, optically transparent, and easily processing material [14]. This polymer is greatly employed for microfluidics and flexible affinity sensing platform [15], due to many advantages, such as miniaturization aimed at reducing the amount of samples and reagents, minimize time and cost, improve reproducibility, automate separations and detections. Different microfluidic platforms based on PDMS have been proposed to provide microchannels or microchambers for purification, processing and sensing of analytes, to achieve separation and detection with high resolution and sensitivity [15]. Moreover, PDMS is recognized as a highly biocompatible material [16,17], which has the intrinsic capacity of spontaneously and strongly adsorb nucleic acids [18,19]. This effect becomes more evident when PDMS surfaces are endowed with positive charges [20], a modification easily performed due to the capability of this material to undergo functionalization with silane groups [21]. Not only the functionalization of PDMS is easily obtained, but also PDMS itself can be used to introduce novel properties on other polymeric materials [22].

Our group already demonstrated the huge potentialities of PDMS-based microdevices for the purification and analysis of biomarkers [11,19]. Here, two configurations of spiral-shaped microdevices are presented. The two configurations have different geometries, which display different surface to volume $(\mathrm{S} / \mathrm{V})$ ratios. This aspect is particularly important since a microfluidic device with higher $\mathrm{S} / \mathrm{V}$ could adsorb higher amounts of biomarkers and, possibly, capture a higher amount of rare microRNAs. The capture of microRNAs occurs on the microdevice surfaces, which are functionalized with positive charges able to attract the negative charges present on the phosphate groups of the microRNA backbones. After an extensive study of PDMS properties in adsorbing/eluting microRNAs, the best conditions were transferred to two types of microfluidic devices, which were deeply characterized. The best working conditions were then validated via real-time PCR for the detection of a pool of microRNAs related to non-small cell lung cancer (NSCLC), selected as proof-of-principle of a biological application.

\section{Materials and Methods}

\subsection{Materials}

(3-Aminopropyl)trimethoxysilane (APTMS), trichlorosilane and ethanol 99.8\% were acquired from Sigma-Aldrich. 2-[Methoxy-(polyethyleneoxy)pro-pyl]trimethoxysilane, tech-90, with 6-9 $\mathrm{C}_{2} \mathrm{H}_{4} \mathrm{O}$ units, called PEG-s, was acquired from Fluorochem (UK), while polydimethylsiloxane (PDMS) (SYLGARD ${ }^{\circledR} 184$ elastomer) was obtained from Dow Corning Corporation (USA). ThermoFisher Scientific supplied the TaqMan ${ }^{\circledR}$ MicroRNA Reverse Transcription Kit, the TaqMan ${ }^{\circledR}$ MicroRNA Assay-hsa-20a, 222 and 320, the TaqMan ${ }^{\circledR}$ Universal PCR Master Mix. Synthetic fluorescently-labelled hsa-miR-1246 (5'-TAMRA-AAUGGAUUUUUGGAGCAGG-3', herein named miR-1246-TAMRA), as well as synthetic miR-20a (5'-UAAAGUGCUUAUAGUGCAGGUAG-3'), miR-222 (5'-AGCUACAUCUGGCUACUGGGU-3') and miR-320 (5'-AAAAGCUGGGUUGAGAGGGCGA-3') were purchased from IDT Integrated DNA Technologies (Belgium). Distilled DNAse/RNAse free water (named water herein) was acquired from Gibco ${ }^{\mathrm{TM}}$ (USA). Finally, the APL 1 buffer derived from the QIAamp UCP Pure Pathogen Blood kit and called "lysis buffer", was provided by Qiagen (Germany).

\subsection{PDMS Planar Surfaces Fabrication}

PDMS flat surfaces were produced by spin coating [20]. Briefly, 10 parts of PDMS base were mixed with 1 part of its curing agent, the mixture was hand stirred for $5 \mathrm{~min}$ and subsequently degassed for $30 \mathrm{~min}$ under vacuum until bubble-free. Silicon substrates $\left(1 \times 1 \mathrm{~cm}^{2}\right)$ were employed as support. Silicon dice underwent an argon plasma treatment of $2 \mathrm{~min}$ at 2 mbar with an RF coil applied power of 
$10.5 \mathrm{~W}$ to remove organic contaminants and hydroxylate the surface, thus allowing PDMS to better adhere. Silicon substrates were subsequently coated with PDMS by means of a spin coater (Model WS400B-6NPP/LITE, Laurell Technologies Corporations, North Wales, PA, USA) using the following protocol: $5 \mathrm{~s}$ at $2000 \mathrm{rpm}$ and $1 \mathrm{~min}$ at $6000 \mathrm{rpm}$. Finally, surfaces were cured in a convection oven (BINDER Inc., Tuttlingen, Germany) at $110^{\circ} \mathrm{C}$ for two hours to allow PDMS polymerization.

\section{3. $p H$ Dependent Functional Assay on Amino-Silanized PDMS Planar Surfaces}

PDMS planar surfaces were functionalized with a silane mixture (named AS), containing $0.1 \% \mathrm{v} / \mathrm{v}$ APTMS and $0.9 \%$ v/v PEG-s silane through a wet functionalization procedure. Prior to silanization, in order to remove contaminants and favor the exposure of their hydroxyl groups, PDMS substrates were treated with an argon plasma for 2 min at 2 mbar with an RF coil applied power of $10.5 \mathrm{~W}$. Shortly after plasma treatment, PDMS surfaces were incubated with the organosilane mixture dissolved in absolute ethanol in a hermetic glass kettle for $10 \mathrm{~min}$ at $60^{\circ} \mathrm{C}$. After the reaction, the PDMS surfaces were rinsed three times in ethanol and dried with nitrogen.

To assess the ability of PDMS surfaces to adsorb and release microRNAs, a functional microRNA adsorption assay with synthetic TAMRA-conjugated miRNA mimics was performed. A total of $10 \mathrm{ng}$ of miR-1246-TAMRA dissolved in buffers at different $\mathrm{pH}$ but with the same ionic strength (i.e., $20 \mathrm{mM}$ ) or dissolved in water, were incubated on the surface for $20 \mathrm{~min}$ at room temperature to allow the optimal adsorption of miRNA. After incubation, the unbound material was removed and the surfaces underwent three washing steps with the same buffer as incubation. The remaining microRNA adsorbed by the surface was visualized and analyzed by fluorescence microscopy using a Leica DMLA (Leica Microsystems, Germany), equipped with a mercury lamp and fluorescence filter L5 (Leica Microsystems, Germany). All substrates were observed with a 20× magnification objective and measured with a cooled charge-coupled device (CCD) camera (DFC 420C, Leica Microsystems, Germany). After imaging, surfaces were subjected to a release step by incubating with buffers either at $\mathrm{pH} 5,7$ or 9 (all with $20 \mathrm{mM}$ ionic strength) for $10 \mathrm{~min}$ at room temperature. At the end of this step, the released material was removed and surfaces were imaged again as described before. For both imaging, i.e., after adsorption and after release of microRNAs, sampling was performed at least in triplicates for each surface and images were analyzed with Fiji software [23].

\subsection{PDMS Microdevices Fabrication}

The two types of microfluidic devices were fabricated as follows: an upper PDMS reaction chamber was obtained by mold-casting and subsequently bonded on a silicon support coated by a thin layer of PDMS. The reaction chamber was designed (Figure 1, Panels (a) and (b)) by a 3D computer aided design (CAD) software (Rhinoceros ${ }^{\circledR}$ ) and molded over a spiral shaped template fabricated with SU-8 photolithography on a silicon wafer support (Figure 1, Panel (c)). In details, the first spiral device (from now called Chip A) was designed with a squared channel with section of $200 \mu \mathrm{m}$ wide and $200 \mu \mathrm{m}$ deep, with walls spacing width of $200 \mu \mathrm{m}$. The second device (from now called Chip B) was defined by a rectangular cross section $60 \mu \mathrm{m}$ wide and $200 \mu \mathrm{m}$ deep with walls spacing $150 \mu \mathrm{m}$ wide. The total length of the spiral channel differs for the two microfluidic devices, being $353 \mathrm{~mm}$ for Chip A and $1078 \mathrm{~mm}$ for Chip B. The spiral channel is connected with the exterior by two inlet/outlet holes.

The fabrication of PDMS microfluidic devices started with the preparation of the SU-8 mold, as explained below. Firstly, silicon wafer support was coated by SU-8 (Microchemicals GmbH) using a spin coater (Spinner 150 WAFER SPINNER) by setting $10 \mathrm{~s}$ at $500 \mathrm{rpm}$ and $60 \mathrm{~s}$ at $2000 \mathrm{rpm}$. Then a soft bake process in two thermal steps was performed: the first step at $65{ }^{\circ} \mathrm{C}$ for $8 \mathrm{~min}$ and the second at $95{ }^{\circ} \mathrm{C}$ for $80 \mathrm{~min}$, both on the hot plate. The SU-8 was exposed for $30 \mathrm{~s}$ to standard UV photolithography by means of a double side mask aligner (Neutronix Quintel NXQ 4006) used in contact mode, ensuring the correct alignment between the SU- 8 and the desired mask patterns. The post bake was characterized again by two thermal steps: the first at $65^{\circ} \mathrm{C}$ for $5 \mathrm{~min}$ and the second at $95^{\circ} \mathrm{C}$ for $25 \mathrm{~min}$, on a hot plate. After that, the SU-8 was developed using Propylene glycol methyl ether 
acetate (PGMEA) for $20 \mathrm{~s}$ to obtain the finished mold. Finally, the released SU-8 structures were hard baked at $170^{\circ} \mathrm{C}$ for $15 \mathrm{~min}$. To guarantee the correct detachment of PDMS from the mold, a silanization step with trichloromethylsilane $\left(\mathrm{CH}_{3} \mathrm{Cl}_{3} \mathrm{Si}\right)$ and toluene in a 1:10 ratio was performed on mold surface. Before silanization, the SU-8 mold was subjected to an $\mathrm{O}_{2}$ plasma treatment ( $300 \mathrm{~W}, \mathrm{O}_{2} 30 \%, 1 \mathrm{~min}$ ). Next, mold surfaces were silanized through a wet phase for $4 \mathrm{~h}$, then rinsed with 2-propanol and dehydrated on a hot plate at $170{ }^{\circ} \mathrm{C}$ for $10 \mathrm{~min}$.

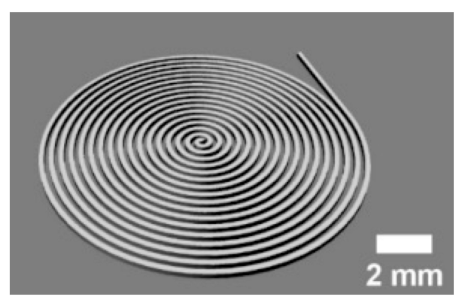

(a)

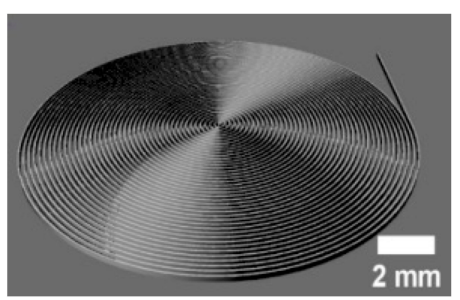

(b)

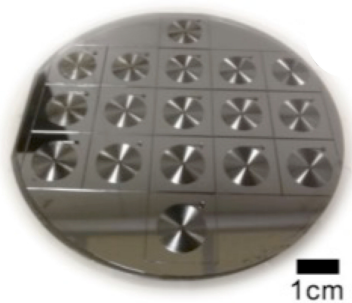

(c)

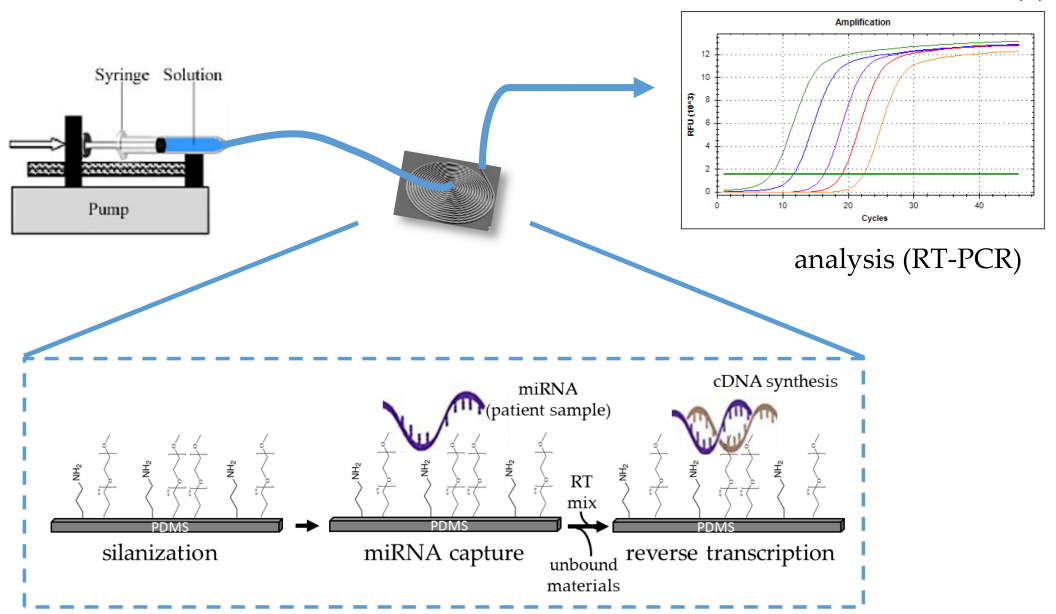

(d)

Figure 1. Computer aided design (CAD) representation of the channels featured in the two types of microdevices (Chip A in Panel (a) and Chip B in Panel (b)) used in this study. Panel (c): a wafer before dicing in single microdevices. Panel (d): experiment workflow. The microdevice is placed in a proper holder with inlet tube fed by a microsyringe pump and outlet tube to collect cDNA for RT-PCR analysis.

To perform the mold casting of the reaction chamber, PDMS was prepared by hand mixing the pre-polymer and the curing agent in a 10:1 ratio, followed by a degassing step after which PDMS was poured on the mold. Curing reaction was performed at $120^{\circ} \mathrm{C}$ for $15 \mathrm{~min}$. The freshly cured spiral chambers were cleaned with acetone and 2-propanol, and dehydrated on a hotplate at $70{ }^{\circ} \mathrm{C}$ for $5 \mathrm{~min}$. In the meantime, the silicon wafer acting as support was pre-cut, cleaned with sulphuric acid and hydrogen peroxide solution ( $3: 1 \mathrm{v} / \mathrm{v}$ ratio) for $5 \mathrm{~min}$, rinsed 3 times in water and dried with nitrogen. The silicon support was coated with a thin layer of PDMS by means of a spin coater (Spinner 150 WAFER SPINNER) with the following protocol: $5 \mathrm{~s}$ at $500 \mathrm{rpm}$ and $60 \mathrm{~s}$ at $3000 \mathrm{rpm}$ and cured at $120^{\circ} \mathrm{C}$ on a hot plate for $5 \mathrm{~min}$. The spiral chambers were bonded on the silicon support by means of a standard $\mathrm{O}_{2}$ plasma treatment $\left(300 \mathrm{~W}, \mathrm{O}_{2} 60 \%, 2 \mathrm{~min}\right)$. Once assembled, microfluidic devices were put at $90^{\circ} \mathrm{C}$ for an hour in the oven. Figure 1 shows the PDMS microdevices and a scheme of experiment workflow. 


\subsection{Microdevices Characterization}

The microdevice cross sections were characterized with a field emission scanning electron microscopy (Zeiss Supra 40 FE-SEM) to check the quality of microchannels. The PDMS microdevices were cut perpendicular to the pattern and then metalized with Chromium to allow the FE-SEM characterization.

The chemical characterization was carried out by X-ray photoelectron spectroscopy (XPS). XPS measurements were performed using a Kratos Axis Ultra DLD instrument (Kratos Analytical, Manchester, UK) equipped with a hemispherical analyzer and a monochromatic $\mathrm{Al} \mathrm{K} \alpha(1486.6 \mathrm{eV})$ $\mathrm{X}$-ray source, in spectroscopy mode. PDMS planar surface and microdevices were analyzed with a take-off angle between the analyzer axis and the sample surface of $0^{\circ}$, corresponding to a sampling depth of approximately $10 \mathrm{~nm}$. For each microdevice sample, a survey (in the $1300,-5 \mathrm{eV}$ energy range) was recorded inside and outside channels in order to check for the presence of impurities derived from the fabrication process. Charge compensation was achieved using a charge neutralizer located at the bottom of the electrostatic input lens system. Survey spectra were aligned setting $C$ 1s core level peak at $284.38 \mathrm{eV}$, the PDMS value in the literature [24]. The quantification, reported as relative elemental percentage, was carried out extracting core levels from the survey spectra. All XPS data were analyzed using the software described in Speranza and Canteri [25].

\subsection{Microdevices Functionalization and Set up of Working Conditions}

Prior to silanization, the internal surfaces of the PDMS microdevices were treated with an argon plasma for $2 \mathrm{~min}$ at 2 mbar with an RF coil applied power of $10.5 \mathrm{~W}$. Shortly after plasma treatment, PDMS microdevices were placed in a proper holder and inlet and outlet tubes were inserted (Figure 1, Panel (d)). The inlet tube was connected to a microsyringe (Legato 185, KD Scientific, Holliston, MA, USA), to deliver liquids with specific flow rates in all steps of this work. Organosilane mix, made of $0.1 \% \mathrm{v} / \mathrm{v}$ APTMS and $0.9 \% \mathrm{v} / \mathrm{v}$ PEG-s diluted in ethanol, was fluxed at $10 \mu \mathrm{L} / \mathrm{min}$ to fill the microdevice. Once full, the microdevice was placed in a water bath at $60{ }^{\circ} \mathrm{C}$ and the mix was continuously fluxed at $2 \mu \mathrm{L} / \mathrm{min}$ for 5 additive minutes. In setup experiments, the silanization time was changed in order to find the optimal conditions. The excess of organosilanes mixture was then removed by fluxing 10 volumes of ethanol followed by 10 volumes of water, both at $25 \mu \mathrm{L} / \mathrm{min}$. Flux rate of microRNAs insertion and washing were optimized by changing the microsyringe parameters.

\subsection{On-Chip microRNAs Functional Assay and Real-Time PCR}

To explore the microdevices performances, a functional assay with synthetic miRNA-1246-TAMRA was setup. Briefly, the fluorescently-labelled miR-1246 spiked either in water or human plasma, was inserted in the microdevice, normally at $10 \mu \mathrm{L} / \mathrm{min}$ flow rate. After a wash with water at $1 \mu \mathrm{L} / \mathrm{min}$ for 10-15 min, the adsorbed microRNA was imaged either by fluorescence or confocal microscopy. For the fluorescence microscopy imaging, a Leica DMLA microscope (Leica Microsystems, Wetzlar, Germany), equipped with a mercury lamp and fluorescence filter L5 (Leica Microsystems, Wetzlar, Germany) was employed. All microdevices were observed with a $2.5 \times$ magnification objective and measured with a cooled CCD camera (DFC 420C, Leica Microsystems, Wetzlar, Germany). To acquire images covering the whole chip, the microscope motorized stage was controlled through a BeanShell script running in MicroManager (version 1.4.23, micro-manager.org), acquiring 54 tiles for each image. The reconstruction of images as well as all the analyses were performed with the Fiji software [23] and Fiji custom macros. The confocal imaging was performed with a Leica SP5-II confocal microscope (Leica Instruments, Wetzlar, Germany), equipped with a helium/neon $(543 \mathrm{~nm})$ laser. Samples were observed utilizing a $20 \times$ objective, while to help the microRNAs visualization on walls, microdevice channels were cut at $30^{\circ}$. To acquire the TAMRA signal, fluorophores were excited with the helium/neon laser, using an emission detector wavelength range from 570 to $660 \mathrm{~nm}$.

On-chip miRNA purification and detection was validated with synthetic miRNAs dissolved in water or in blood plasma. Purification of synthetic microRNAs was performed by spiking synthetic 
microRNAs ranging from $0.1 \mathrm{pg}$ to $1 \mathrm{ng}$ either in water or in human plasma mixed in a 1:1 ratio with lysis buffer (APL1 buffer, Qiagen, Germantown, MD, USA). To obtain human plasma, blood samples were collected from healthy donors into EDTA-treated collection tubes. For plasma preparation, fresh blood was centrifuged at $700 \mathrm{~g}$ for $10 \mathrm{~min}$ to pellet the cell fraction. The supernatant was then collected avoiding contact with the buffy coat. Before using, plasma samples were centrifuged at $1000 \mathrm{~g}$ for 5 min at $4{ }^{\circ} \mathrm{C}$ to pellet the debris.

The proper amount of synthetic microRNA diluted either in water or in plasma was inserted in the microdevice at room temperature with the microsyringe at $10 \mu \mathrm{L} / \mathrm{min}$ for a suitable time (10-20 min). During this step, the microRNA is adsorbed on the amino-silanized surfaces of microdevice channels. To remove the excess of microRNAs, a wash with water was performed at $1 \mu \mathrm{L} / \mathrm{min}$ for 10-15 min.

For the on-chip analysis of miRNAs, a two-steps protocol based on reverse transcription coupled with real-time PCR was employed. Reverse transcription (RT) was performed directly on-chip on the miRNAs adsorbed by the PDMS surface by adding the RT master mix at $10 \mu \mathrm{L} / \mathrm{min}$ into the microdevice. TaqMan ${ }^{\circledR}$ MicroRNA Reverse Transcription Kit included $100 \mathrm{mM}$ dNTPs (with dTTP), MultiScribe $^{\mathrm{TM}}$ Reverse Transcriptase (50 U/ $\left.\mu \mathrm{L}\right), 10 \times$ Reverse Transcription Buffer, RNase Inhibitor $(20 \mathrm{U} / \mu \mathrm{L}), 20 \times$ miR-specific RT primers. The reaction was carried out for $30 \mathrm{~min}$ at $16{ }^{\circ} \mathrm{C}, 30 \mathrm{~min}$ at $42{ }^{\circ} \mathrm{C}$, and $5 \mathrm{~min}$ at $85^{\circ} \mathrm{C}$ on a XP Thermal cycler (Bioer Technology Co., Ltd.) equipped with a flat thermal block. Once synthetized, cDNA was subsequently recovered from the microdevice with the help of microsyringes and amplified with TaqMan ${ }^{\circledR}$ MicroRNA Assay-hsa-miR-specific in standard conditions. An amount of $20 \mu \mathrm{L}$ of real-time PCR mix were composed of TaqMan ${ }^{\circledR}$ Small RNA Assay $(20 \times)$, TaqMan ${ }^{\circledR}$ Universal PCR Master Mix II (2×), nuclease-free water and cDNA, following the manufacturer's instructions. The cycling protocol, $95^{\circ} \mathrm{C}$ for $10 \mathrm{~min}$ and 45 cycles of $95^{\circ} \mathrm{C}$ for $15 \mathrm{~s}$ and $1 \mathrm{~min}$ at $60^{\circ} \mathrm{C}$ was run on a CFX Connect ${ }^{\mathrm{TM}}$ Real-Time PCR Detection System (BioRad). Samples were amplified in triplicates and non-templates controls were included (amplification negative control). Data were analyzed with BioRad CFX Manager 2.1 software and threshold cycles $(\mathrm{Ct})$ were reported and compared with a standard curve, build with known amounts of cDNA, for quantification.

\section{Results and Discussion}

The direct adsorption of microRNAs on a PDMS microdevice was already demonstrated by our group $[19,20]$. The overall handling, however, was manually driven and therefore further investigations were needed in order to automatize and improve the performances of the microdevice. In a perspective of use in clinically relevant environments, the partial or total automatization of a microdevice could indeed make the difference between research purposes or real-life applications.

\subsection{Setup of the Optimal Conditions for microRNAs Adsorption on PDMS Planar Surfaces}

PDMS planar surfaces were selected to mimic the surfaces of microdevice channels with the aim of studying the best $\mathrm{pH}$ conditions for microRNAs capture and release. The interaction between primary amines coating surfaces and nucleic acid is indeed well documented [26,27]. Since the main driving force of this phenomenon relies on the electrostatic interaction, a change in the $\mathrm{pH}$ of nucleic acid solutions could affect their adsorption. As a first step in the setup of the optimal working conditions, an investigation on the dependence of microRNA adsorption on $\mathrm{pH}$ was therefore undertaken. Moreover, considering the potential use of this microdevice aimed at biomarker detection, the synthetic miR-1246 was selected because of its suggested potential role as biomarker for several cancer types [28,29], including the non-small cell lung cancer (NSCLC) [30].

Synthetic miR-1246 conjugated with the fluorescence dye TAMRA was diluted in buffers with $\mathrm{pH}$ ranging from 4 to 9 , while the ionic strength was kept constant at $20 \mathrm{mM}$ to minimize the possible interference of counter-ions or ionic bridges. The fluorescent microRNA was incubated on the silanized PDMS surfaces. After adsorption and washing, the surfaces were imaged with a fluorescence microscope. The adsorption of microRNAs was higher at $\mathrm{pH}$ around 5 , but a quite good adsorption was observed also at neutral pH (Figure 2, Panel (a)), confirming our previous findings related to 
the behavior of amino-silanized surfaces [27]. Interestingly, the highest adsorption (fluorescence signal $=26.4 \times 10^{3} \pm 180$ ) was obtained when the microRNA was diluted in water (i.e., very low ionic strength, $\mathrm{pH}$ slightly acidic). This behavior could be easily explained considering the net charge of the interacting molecules. In fact, RNA molecules are negatively charged under physiological conditions, due to the presence of charged phosphate groups along the backbone of the nucleic acid. Under the same conditions, the majority of the amino groups are in their protonated forms, as discussed below, so the APTMS exposes a positive surface, prone to attract negative molecules. Instead, at basic $\mathrm{pH}$ the amino groups are mostly in their un-protonated form, thus the APTMS surface becomes neutral. Moreover, the presence of PEG-s molecules helps the uniform distribution of positive charges, as demonstrated by our group [19,31].

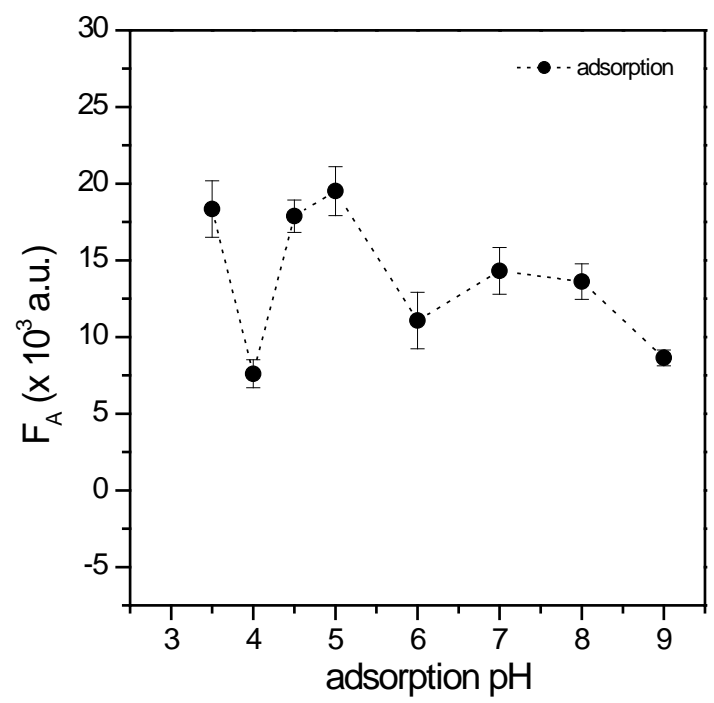

(a)

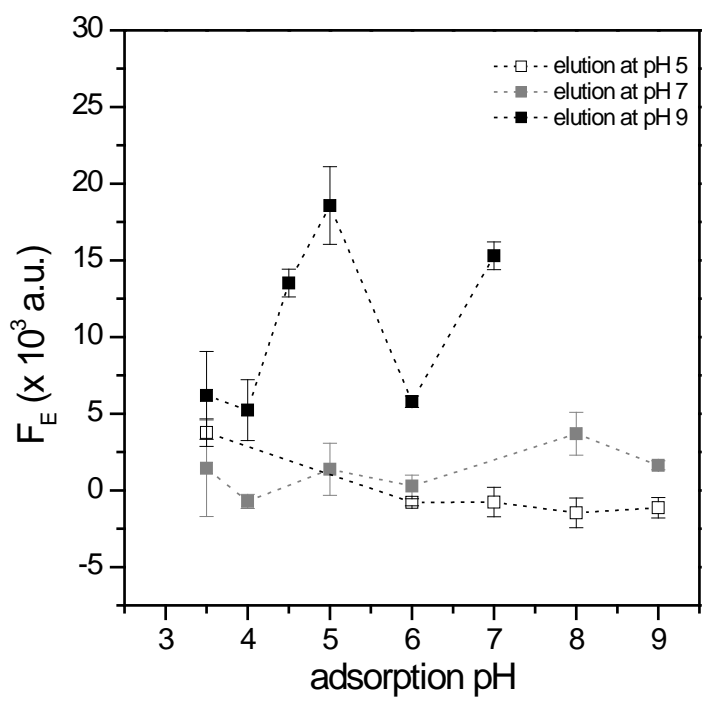

(b)

Figure 2. MicroRNA adsorption (Panel (a)) and elution (Panel (b)) from flat polydimethylsiloxane (PDMS) surfaces as a function of $\mathrm{pH}$. Panel (a): $10 \mathrm{ng}$ of synthetic miR-1246, conjugated with the florescent dye TAMRA, were incubated on the silanized surfaces at different $\mathrm{pH}$. After three washes with the same buffer as for incubation, the fluorescence of the adsorbed microRNA was measured $\left(\mathrm{F}_{\mathrm{A}}\right)$. Panel (b): fluorescence of the eluted microRNA $\left(\mathrm{F}_{\mathrm{E}}\right)$. The microRNA adsorbed at different $\mathrm{pH}$ was eluted from the surfaces with buffers at three different $\mathrm{pH}$ values: $\mathrm{pH}=5$ (white squares), $\mathrm{pH}=7$ (gray squares) and $\mathrm{pH}=9$ (black squares). After elution, surfaces were imaged and the resulting signal was subtracted from $\mathrm{F}_{\mathrm{A}}$, obtaining values of the eluted microRNA $\left(\mathrm{F}_{\mathrm{E}}\right)$. Data are means of at least three independent replicates, while error bars represent standard errors.

Once adsorbed, the release of microRNA was also investigated as a function of $\mathrm{pH}$. The $\mathrm{pK}$ of a typical organic primary amine in solution ranges around 10-11 units [32]; but, when molecules carrying primary amines are covalently bound to a surface, the $\mathrm{pK}$ of such amines could be lowered until around 6.5 on gold surfaces [33], and around 4 on silicon oxide [34]. To verify to which extent the amine groups attached to PDMS surfaces (amino-silanized PDMS surfaces object of this study) are influenced by such effect, three $\mathrm{pH}$ conditions were selected for the releasing step, i.e., $\mathrm{pH}$ 5, 7 and 9 (Figure 2, Panel (b)). As expected, the worst result is obtained for elution at $\mathrm{pH} 5$, but also elution at $\mathrm{pH} 7$ resulted scarce at every adsorption $\mathrm{pH}$ tested. On the contrary, microRNA release was almost complete when the elution step occurred at $\mathrm{pH}$ 9, i.e., well over the above reported values of surface-bound amines $\mathrm{pK}$. We consider then that the lowering of the $\mathrm{pK}$ value of amines attached to PDMS, if ever happens, is negligible in our conditions. Vezenov et al. [35] suggest indeed that the pK shift may be connected to the presence of a hydrophobic environment surrounding the amine groups. Our formulation, embedding APTES with PEG molecules, may mitigate this effect. Notably, elution 
at $\mathrm{pH} 9$ was complete also when the microRNA adsorption step occurred in water (the measured fluorescence value was $25.7 \times 10^{3} \pm 180$ ).

Experiments run on PDMS planar surfaces took to the conclusion that best conditions for microRNA adsorption are the dilution in water followed by elution at $\mathrm{pH}$ 9. Importantly, the adsorption at physiological $\mathrm{pH}$ is also high, allowing to process biological samples without changing the $\mathrm{pH}$ of solution before microRNA capture.

\subsection{Characterization of PDMS Spiral-Shaped Microdevices}

Prior to analyze the performances of spiral-shaped microdevices for the capture of microRNAs, a morphological and chemical characterization was performed. Several microdevices were sectioned and measured at the Field Emission Scanning Electron Microscope (FESEM) (Figure 3) in order to check for the real dimensions of microdevice channels. For Chip A, channels were $182 \pm 13 \mu \mathrm{m}$ wide and $325 \pm 88 \mu \mathrm{m}$ deep, with a mean volume of $20 \mu \mathrm{L}$, while for Chip B channels were $60 \pm 4 \mu \mathrm{m}$ wide, $197 \pm 13 \mu \mathrm{m}$ deep and $12 \mu \mathrm{L}$ as total volume. The two microdevices differed for their surface to volume ratio (S/V for Chip A is $18.0 \mathrm{~mm}^{-1}$ and for Chip B is $46.2 \mathrm{~mm}^{-1}$ ).

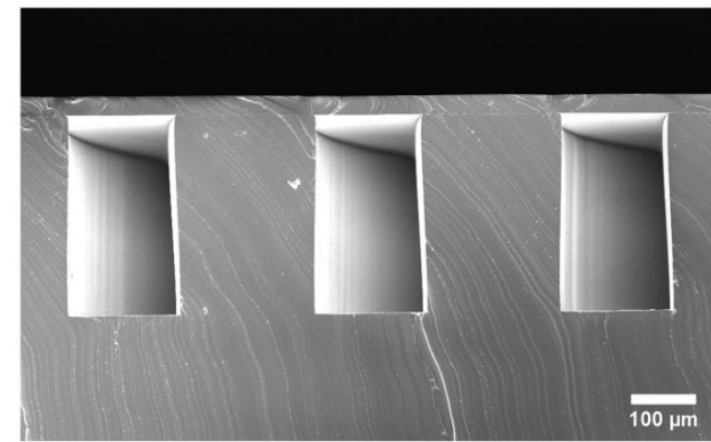

(a)

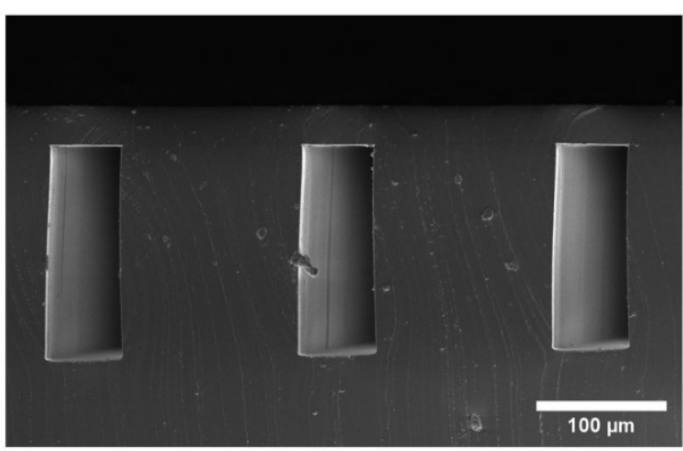

(b)

Figure 3. FESEM images of microdevice A (Panel (a)) and B (Panel (b)). Microdevices were cut to expose microdevice channels. White bars represent $100 \mu \mathrm{m}$.

The chemical composition of the microdevice inner surfaces was also analyzed and compared to bulk PDMS (i.e., freshly-cut surfaces). During the replication process, in order to easily detach the PDMS, the mold was coated by a trichloromethylsilane anti-adhesion layer, which was deposited by a wet phase deposition. Since this reagent could contaminate the inner surface of channels of the PDMS replica, and possibly interfere with the downstream biological applications, a thorough XPS analysis and a PCR-compatibility assay were performed.

It is indeed well known that materials composing microdevices and residues of reagents employed in their processing, can inhibit microdevice performances, in particular when specific amplification of nucleic acids is desired, as in this case [36,37]. The presence of chlorine, deriving from trichloromethylsilane, was investigated via XPS. XPS survey spectra taken inside a microdevice channel and on bulk PDMS material (Figure 4) showed the presence of oxygen, carbon and silicon, as expected for PDMS, while no chlorine was detected. The quantification of elements, reported as relative elemental percentage in Table 1, revealed a similar composition for all the measured samples. Similar amount of elements are present both on inner channel surfaces and on bulk microdevice material, as well as on PDMS planar surface and no chlorine was detected in all the tested conditions. The overall elemental composition highlighted by XPS measurements reflects indeed the molecular composition of PDMS $\left[\left(\mathrm{C}_{2} \mathrm{H}_{6} \mathrm{OSi}\right) \mathrm{n}\right]$, with $\mathrm{C} / \mathrm{O}$ and $\mathrm{C} / \mathrm{Si}$ ratios around 1.7 and 2.5, respectively, in agreement with the literature $[17,20,38]$. 


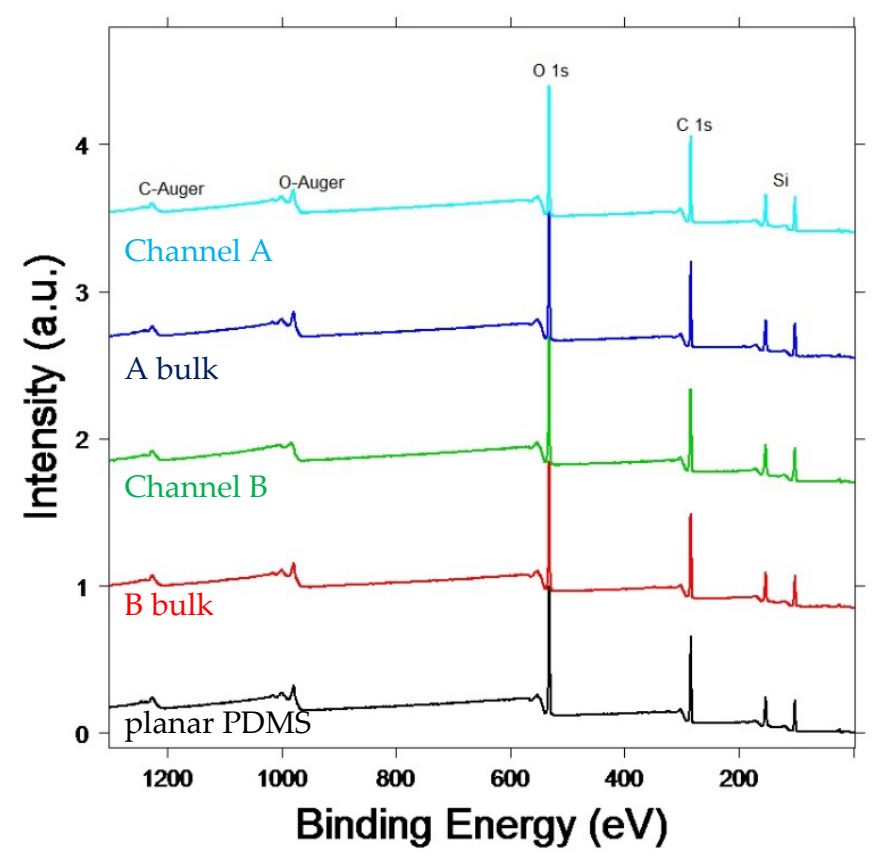

Figure 4. X-ray photoelectron spectroscopy (XPS) survey spectra taken at $0^{\circ}$ take-off angle. Spectra were taken inside microdevices channels (curves named Channel A and Channel B) and outside channels, i.e., on freshly cut microdevice surfaces (A bulk and B bulk). A planar PDMS surface was also measured for comparison (planar PDMS).

Table 1. XPS relative quantification (\%) of $\mathrm{O}, \mathrm{C}$, and $\mathrm{Si}$ detected with a $0^{\circ}$ take-off angle on inner (Channel A or B) and outer surfaces (Chip A or B bulk) of microdevices channels and on PDMS planar surfaces. The standard error does not exceed the $1-2 \%$ of the reported value.

\begin{tabular}{cccc}
\hline Sample & O 1s (\%) & C 1s (\%) & Si 2p (\%) \\
\hline Channel A & 29.4 & 51.7 & 18.6 \\
Chip A bulk & 29.7 & 51.5 & 18.5 \\
Channel B & 29.4 & 49.9 & 20.7 \\
Chip B bulk & 29.9 & 50.1 & 20.0 \\
Planar PDMS & 28.8 & 50.7 & 20.5 \\
\hline
\end{tabular}

Beside XPS measurements, a PCR was performed in order to verify the compatibility of the microdevice material with an amplification reaction. Small pieces of microdevice exposing the channel surface were submerged in the PCR mix and the reaction was run. PCR products were loaded on an agarose gel (Figure S1) and compared with controls (both tubes without PDMS and tubes containing small pieces of a PDMS microdevice known for its compatibility with PCR $[18,19])$. The microdevice internal surfaces were confirmed as perfectly compatible with the PCR reaction.

\subsection{From PDMS Planar Surfaces to Spiral-Shaped Microdevices}

The setup of a functional assay on PDMS planar surfaces as well as the characterization of spiral-shaped microdevices were detailed in the previous paragraphs. These results, however, cannot be directly utilized to effectively functionalize the microdevices, therefore further experiments were undertaken. In particular, protocols had to be adapted to the automated microdevice manipulation. Firstly, the silanization protocol was arranged in order to provide the best performances in terms of microRNAs adsorption.

The silanization mixture, AS, was injected in the microdevice, which was next immersed in water at $60{ }^{\circ} \mathrm{C}$ for different periods. A time spanning from few minutes to one hour was employed to functionalize the microdevices (Figure 5, Panel (a)), while all other functionalization conditions were kept constant. 
Even at short silanization time, a good adsorption of microRNA was observed, allowing selecting 5 min of silanization as the standard condition for following experiments. This conclusion was reinforced by measuring the unbound microRNA collected from every microdevice, at the spectrophotometer. In addition, in this case, the amount of unbound material from microdevices silanized for 5 min was comparable with those of microdevices silanized for 40 or even for $60 \mathrm{~min}$ (Figure S2).
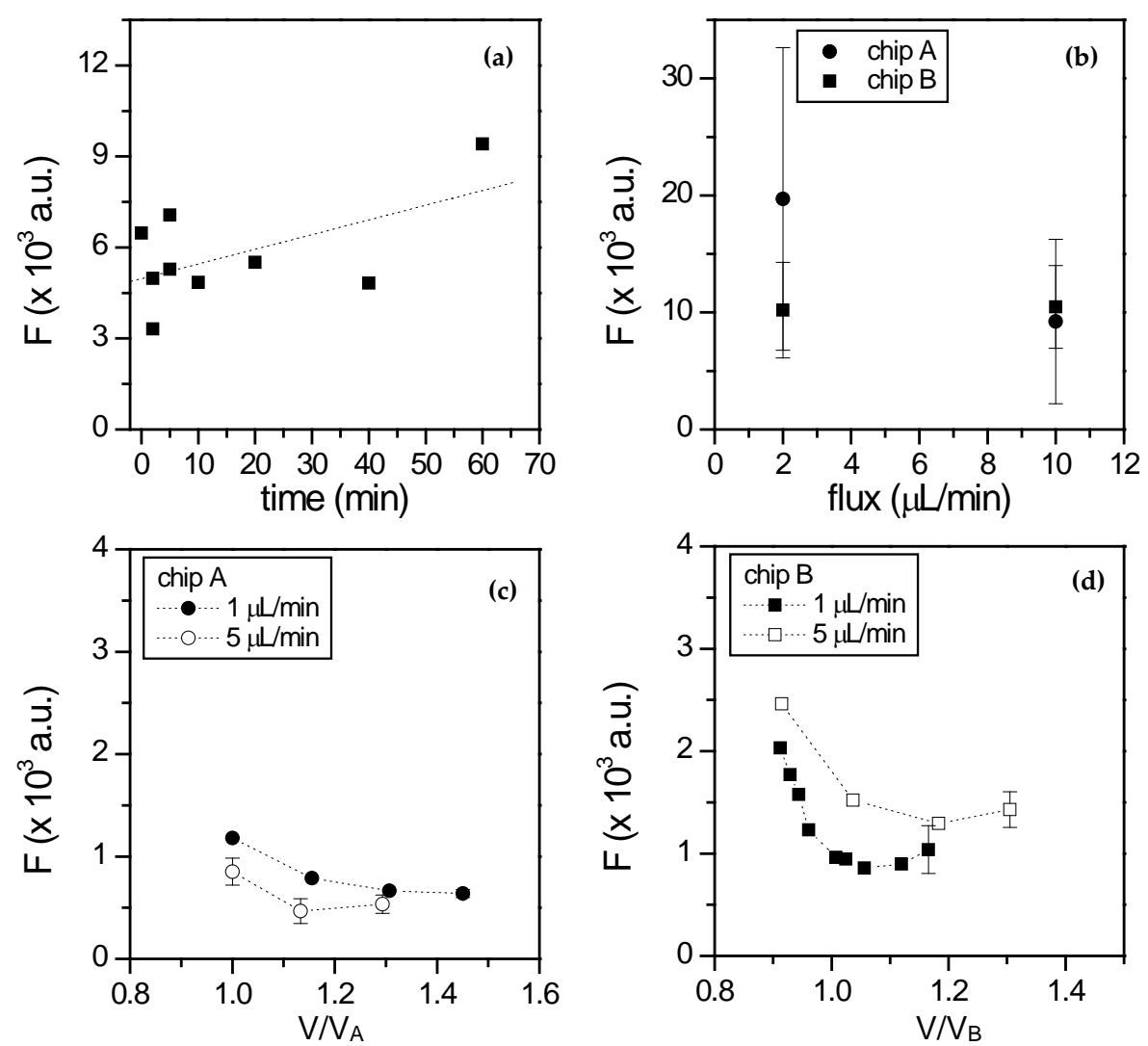

Figure 5. Optimization of protocols from PDMS planar surfaces to spiral microdevices. Fluorescent miRNAs were used to assess functionalization parameters. Panel (a): optimization of silanization time. The microdevice is functionalized with a mixture of 0.1\% APTMS and 0.9\% PEG-s silane (named AS) in immersion at $60{ }^{\circ} \mathrm{C}$ for different times. An amount of $600 \mathrm{ng}$ of fluorescent microRNAs diluted in water are then absorbed to microdevice AS surfaces and the resulting fluorescence is reported as a function of the silanization time. Panel (b): optimization of the flux speed during microRNA insertion. $3 \mathrm{ng} / \mu \mathrm{L}$ miR-1246-TAMRA was inserted either at $2 \mu \mathrm{L} / \mathrm{min} \times 100 \mathrm{~min}$ or $10 \mu \mathrm{L} / \mathrm{min} \times 20 \mathrm{~min}$. Panels $(\mathbf{c}, \mathbf{d})$ : optimization of washes speed after miR adsorption. A total of $600 \mathrm{ng}$ of miR-1246-TAMRA, diluted in human plasma added with lysis buffer, were incubated on microdevices A (Panel (c)) or B (Panel (d)); then a wash with water is performed at different fluxes. Relative volumes are reported (i.e., referred to each chip volume).

Once functionalized, the microdevice is ready to capture microRNAs. Therefore, the next optimization concerned the flux of microRNA insertion, which should be fast enough to allow the adsorption on the microdevice surfaces but not too fast impeding the effective contact of microRNAs with the positively charged surfaces. The same concentration of fluorescent microRNA was inserted in the silanized microdevices (Figure 5, Panel (b)) with two different flow rates for a sufficient time to deliver $600 \mathrm{ng}$ of total microRNA. Both the fluxes gave quite similar results in terms of microRNA adsorption for both the microdevices types (A and B), therefore $10 \mu \mathrm{L} / \mathrm{min}$ was selected as optimal, reducing the insertion time and speeding up the duration of the assay.

Finally, a washing step is needed to remove the unbound material. The microdevice was flowed with water, whose $\mathrm{pH}$ is slightly acidic (see Section 3.1) and therefore the surface charges are not 
affected. Further, in this case, the flow rate is crucial for removing all the unwanted material but preserving all the adsorbed microRNA. Moreover, the volume of water used in the washing step should not exceed the minimum value that is necessary to effectively substitute the channel solution with water, otherwise the equilibrium of microRNA adsorbed on surfaces could be shifted toward its release. Two flux rates were applied either to Microdevice A (Figure 5c) or to Microdevice B (Figure 5d) after the microRNA-capture step. Both fluxes gave similar results, removing the excess of microRNA solution up to a plateau. However, the flux of $1 \mu \mathrm{L} / \mathrm{min}$ for $10 \mathrm{~min}$ was selected as the most efficient in removing the unbound material, while better preserving the adsorbed microRNA.

An injection time of $10 \mathrm{~min}$, i.e., about one washing volume, was judged compatible with the overall duration of the assay, while guaranteeing not to remove the adsorbed microRNA. A scheme visualizing the whole process of microRNA insertion and washing is reported in Figure S3.

In conclusion, moving from planar surfaces to microdevices, the silanization time was reduced to $5 \mathrm{~min}$, the flow rate to insert microRNA was set to $10 \mu \mathrm{L} / \mathrm{min}$, while the washing flow rate was reduced to $1 \mu \mathrm{L} / \mathrm{min}$.

\subsection{Functional Assay for the Characterization of Microdevices for microRNA Adsorption}

Once set up the working parameters for both microdevices, a functional assay aimed at verifying the potentialities of the two microdevices types was carried out. In the previous paragraph, all parameters were set up by measuring the fluorescence signal related to the presence of miR-1246-TAMRA with a wide-field fluorescence microscope. This method is excellent for a relative comparison of the different conditions applied to the microdevices, but results rather poor when a strict quantification is needed. In particular, the collected fluorescence signal is mostly related to the microRNA adsorbed on channels top and bottom surfaces, with a minor contribution from channels lateral surfaces. To check the uniformity of miRNA adsorption on surfaces, a confocal microscope was then used to collect three-dimensional images of the channels for the two kinds of microdevices. Channels of both microdevices were cut diagonally with an angle of about $30^{\circ}$ after microRNA adsorption, to ease the imaging at the confocal microscope (Figure 6).
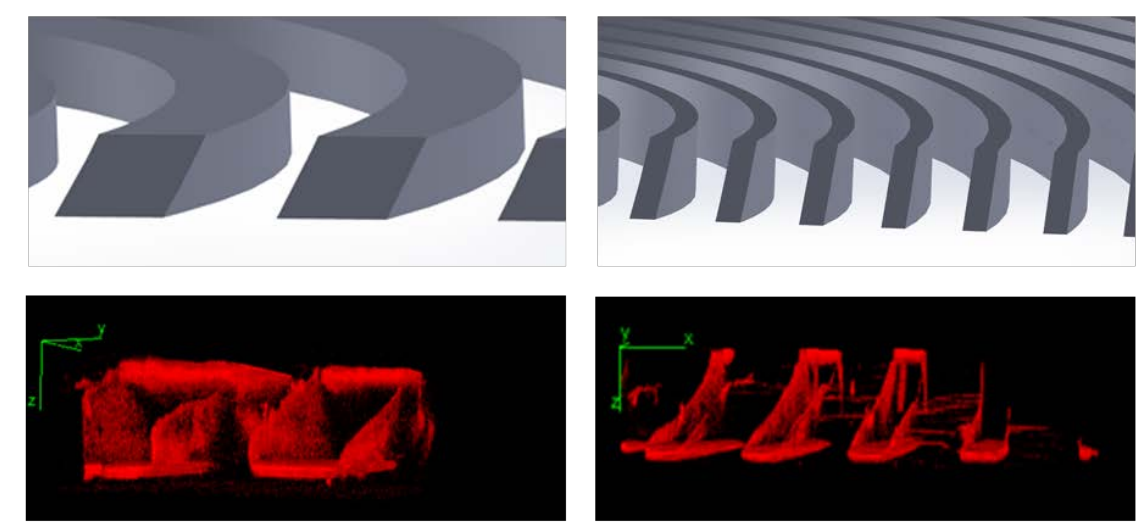

(a)

(b)

Figure 6. Check for surface availability for microRNA adsorption. Panel (a): $1200 \mathrm{ng}$ of miR-1246-TAMRA diluted in water are adsorbed on functionalized microdevice A. Panel (b): $600 \mathrm{ng}$ of miR-1246-TAMRA diluted in water are adsorbed on functionalized microdevice B. After washes, the dry channels were cut diagonally with an angle of about $30^{\circ}$, as depicted on top of each image (CAD representations), and imaged at the confocal microscope in z-stacking mode. Images are 3D reconstructions of microdevice channels.

From data reported in Figure 6, all microdevice surfaces can be considered as properly functionalized and then available for microRNAs capture. The presence of the fluorescent microRNA, 
indeed, is homogenously distributed on all the visible surfaces, confirming the good working conditions previously set up.

Next, we moved to consider the effect of the different total channel surface on the maximum loading capacity of the two kinds of microdevices. In Figure 7, an estimation of the total area covered by fluorescent miRNAs versus the quantity of miRNAs fluxed through the chip is reported. This titration confirmed that Microdevice $B$, which presents a higher total channel surface $\left(S / V \approx 46.2 \mathrm{~mm}^{-1}\right)$, adsorbs a higher amount of microRNA compared to Microdevice $A\left(S / V \approx 18.0 \mathrm{~mm}^{-1}\right)$. For this experiment all other parameters than concentration, i.e., flux rate, microRNA insertion time, flux and washing time, were kept constant. The insertion of $300 \mathrm{ng}$ of pure microRNA saturated the capturing ability of Microdevice A, while a double amount was still well adsorbed by Microdevice B. The total area possibly occupied by microRNA was calculated as $356 \mathrm{~mm}^{2}$ for Chip A and $460 \mathrm{~mm}^{2}$ for Chip B. For Chip A, this value was reached with the adsorption of $300 \mathrm{ng}$ of microRNA, while the double was still adsorbed by Chip B, reflecting the different S/V for the two microfluidic devices. The S/V of Chip B is indeed about the double than S/V of Chip A.

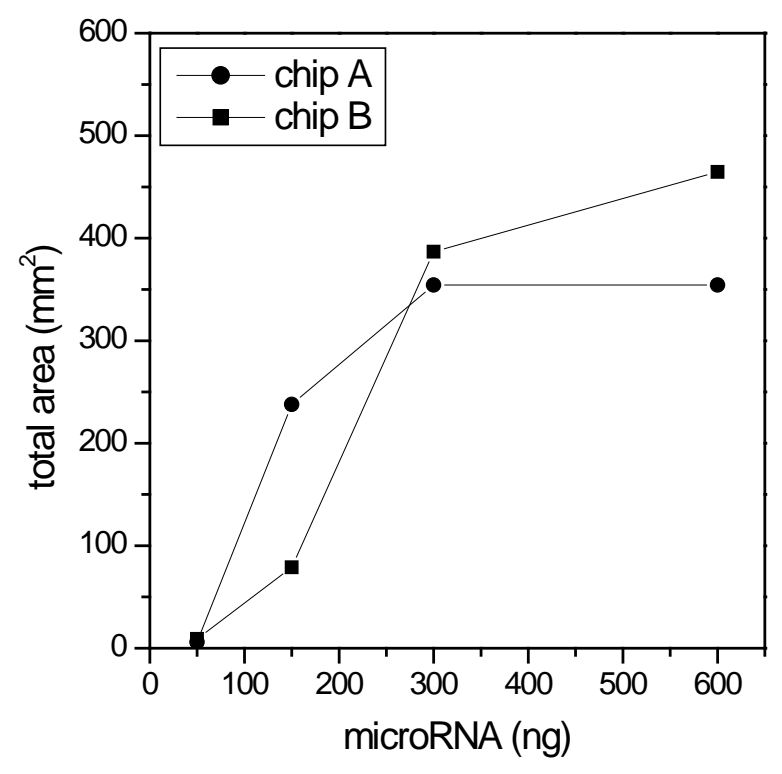

Figure 7. Testing microdevices performances in miRNAs adsorption. The adsorption of different quantities of synthetic fluorescent microRNA (miR-1246-TAMRA) dissolved in water on the two types of chips is measured as the total spiral length occupied by microRNA after washes.

The ability to capture pure microRNA was demonstrated for both microdevices. A further characterization of microdevice performances, more geared toward a possible use of these devices on field, was performed by introducing a constant concentration of fluorescent microRNA spiked in human plasma added with lysis buffer (Figure 8), for different times. The addition of lysis buffer is needed to favor the release of miRNA from vesicles encapsulation or protein complexes, in the perspective of microdevice use with clinically relevant samples. For this analysis, Chip B was selected as the more performant with respect to the Chip A (Figure 7).

Comparing the fluorescence signal intensity that is detected on microdevices fluxed for different times, a linear increment is clearly visible as the fluxing time is increased, as expected. This behavior confirmed that the channel surfaces are able to capture the microRNA even when it is dissolved in a complex matrix, such as human plasma. Moreover, from the same data, one infers that this capability is maintained constant in time, leading to the linear increase of captured miRNAs. Blood plasma, indeed, contains a lot of components (proteins, lipids, small molecules, etc.), which presumably can be adsorbed to the positively-charged surfaces as well as nucleic acids. These components, however, do not impede the further adsorption of microRNA on the surface sites still available. This potentiality 
of PDMS-based microdevice was already demonstrated by our group with a different type of PDMS microdevice with a limited surface available for microRNA capture $[19,20]$. The augmented surface of the here presented microdevices together with their handling automation could help to introduce similar microdevices for the use in real setting.

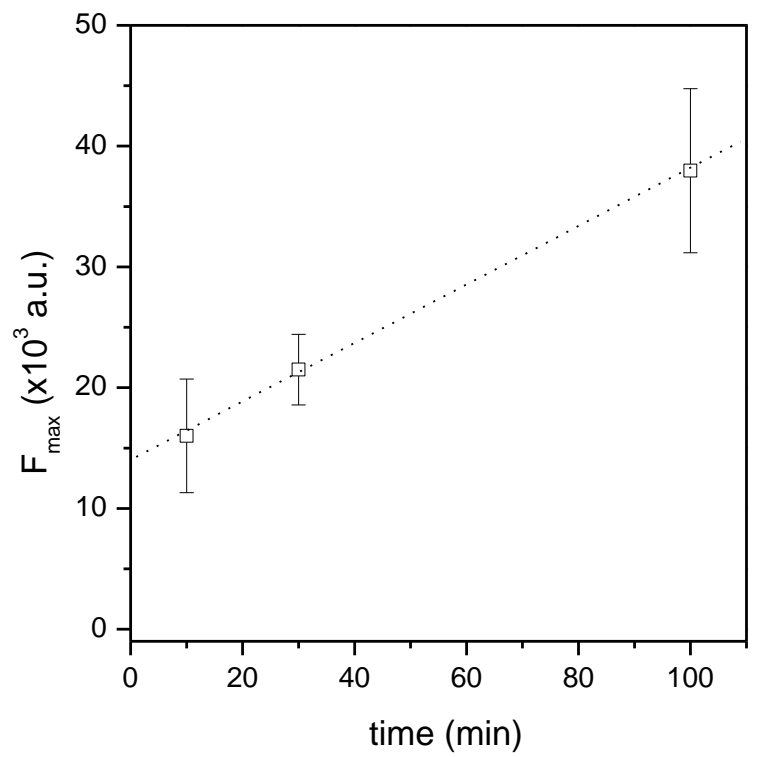

Figure 8. Adsorption of synthetic microRNA on Chip B. The titration of efficiency in capturing microRNAs with time is measured by fluxing miR-1246-TAMRA dissolved in human plasma added with lysis buffer at constant concentration. Fluorescence signal is the mean of maximum signal measured in consecutive coils, while error bars refer to standard error. A linear fit is also shown (dotted line; $\mathrm{R}=0.999$ ).

\subsection{Validation of PDMS-Based Microdevices}

Final application of the PDMS-based microdevices presented in this study is the specific capture of low amounts of microRNAs as biomarkers of oncological pathologies in the context of liquid biopsy. Since the concentration of such circulating biomarkers is quite low, a polymerase chain reaction amplification step is required. The adsorbed miRNAs were directly reverse-transcribed on-chip, then releasing cDNA molecules in solution. This solution was collected to feed the DNA amplification step, using standard tubes and equipment.

A panel of microRNAs often referred as biomarkers of NSCLC $[39,40]$ was selected to validate the microdevices. Either microRNA-20a or microRNA-222 or even microRNA-320 were spiked in water or in human plasma added with lysis buffer and subjected to purification via adsorption on the functionalized microdevices. After the washing step with water to remove the unwanted material possibly adsorbed on channel walls, the proper reverse transcription mix was inserted in each microdevice and the reaction was allowed to proceed directly on-chip on a thermal cycler equipped with a flat thermal block, as previously described [19]. Finally, the solution containing the proper cDNAs was used to feed the RT-qPCR reactions (Figure 9). For all the tested conditions, a decrease in the threshold cycles $(\mathrm{Ct})$ is observed when the amount of spiked microRNA increases. Interestingly, the same amount of spiked microRNA gave different results when spiked in water or in plasma, with a better $\mathrm{Ct}$ in plasma. A possible explanation of this phenomenon relies on the passivation effect due to proteins present in plasma but, obviously, absent when the microRNA is dissolved in water. In other words, the components of a biological sample not only do not adversely compete with microRNA molecules for the adsorption sites, but favor instead their better interaction with the channel surfaces. Same amount of functional sites are initially available on channel surfaces for the two conditions, but in water more anchorage free sites could capture in a constrained conformation the microRNA with 
respect to that in plasma. As a result, this microRNA could be not completely available for the enzyme in the reverse transcription step, yielding a lower amount of cDNA molecules to be amplified via RT-qPCR. Moreover, components of the reverse transcription mix as well as the just-copied cDNA molecules could also be captured on non-passivated chip surfaces.

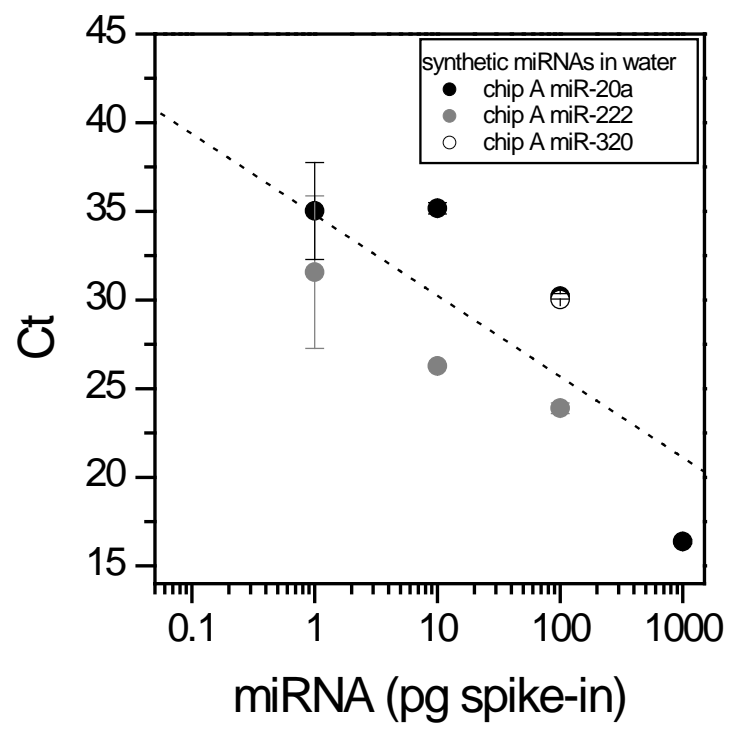

(a)

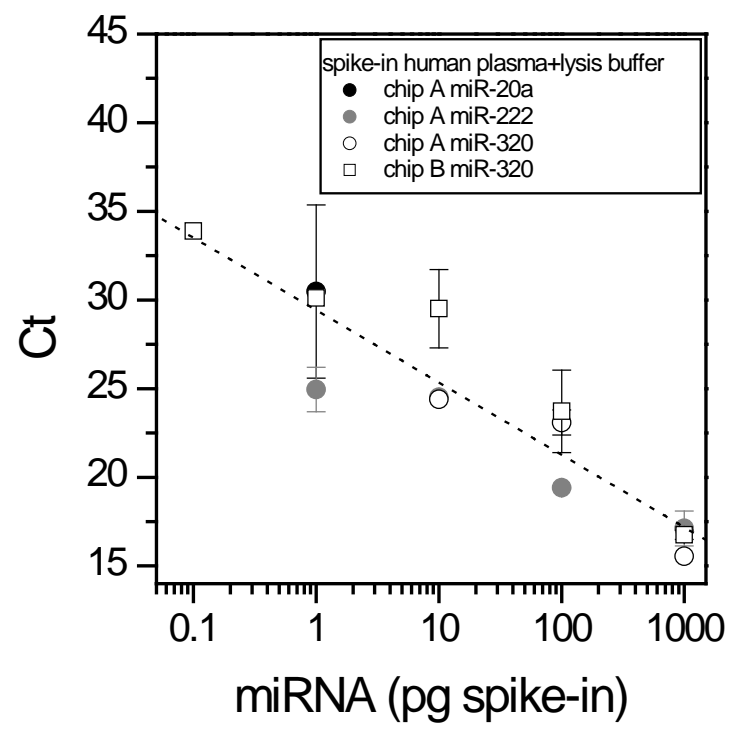

(b)

Figure 9. RT-qPCR results expressed as threshold cycles $(\mathrm{Ct})$ measured when different amounts of synthetic microRNA were spiked in water (Panel (a)) or in blood plasma (Panel (b)). The absorbed microRNA was reverse transcribed directly on-chip, while the cDNA products were amplified in standard conditions. A dotted line, from a logarithmic fit of all data is also shown.

Both in water or in human plasma, the two types of microdevices are able to capture different amounts of synthetic microRNAs, which are directly reverse-transcribed on chip and amplified in standard conditions. As shown in Figure 9, three different microRNAs associated with non-small cell lung cancer were successfully detected at different starting concentrations in spiked water or plasma, confirming no preferences in the adsorption of different sequences. Since synthetic microRNAs were spiked in water or plasma in known concentrations, we treated the data of all three microRNA as similar and fitted all data together (dotted lines in Figure 9). The resulting fit is a straight line (in semi-log graphs) both for miR spiked in water and in plasma confirming the linearity between microRNA incubated and adsorbed on surfaces.

The good performances of microdevices were further tested by detecting microRNAs which are naturally present also in human plasma collected from a healthy donor, even if at lower concentrations. The same protocol as before was applied for the adsorption of miR-20a and miR-222 on Chip A, obtaining a Ct of $31 \pm 2$ and of $32 \pm 4$, respectively. These data were compared with those obtained by using a PDMS microdevice already validated for capturing circulating microRNAs $[19,20]$. This microdevice acts in this context as positive control. The Ct resulting from the capture of miR-20a and miR-222 from the same human plasma sample were $32 \pm 0.1$ and $34 \pm 0.1$, respectively. Therefore, the results of the spiral-shaped microdevice are not only in-line with the positive control but even better. As a conclusion, the spiral-shaped microdevice was demonstrated as a good candidate for the easy detection of microRNAs biomarkers from biological samples, even if a further implementation, especially in terms of fluids manipulation is necessary. Still, the device here presented could be an initial platform for the realization of compact, rapid, non-invasive, easy-to-use and economic tools to be employed in real-settings. 


\section{Conclusions}

The present study shows the fabrication, characterization and functional application of two spiral-shaped microdevices. The PDMS microdevices have different surface to volume ratios and differ for the total internal surface available for the capture of biomarkers. The microfluidic device presenting a higher $\mathrm{S} / \mathrm{V}$ was demonstrated to capture a higher amount of microRNAs. This aspect is especially important since biomarkers, in particular microRNAs, are present in little amounts in body fluids and therefore, a smart method for concentrating and detecting is welcome. The microdevices presented here satisfy this requirement, being able to purify different microRNAs considered as cancer biomarkers from a biological fluid, such as human plasma. The performances of the microdevices approached or even exceeded those of a similar PDMS-based microdevices previously reported in the literature. This consideration fosters the idea of a real use of PDMS-based microdevices as diagnostic tools to identify low abundant microRNA biomarkers in clinical setting.

Liquid biopsy is indeed emerging as a precious source of biomarkers, easily accessible and therefore useful for mass screening and for biomarkers profiling of patients during specific therapies. Microfluidic devices could simplify liquid biopsy, allowing the spread of these methodologies not only for diagnostic purposes, but also for following the progress of a disease. Moreover, the detection of low amounts of biomarkers could take to earlier diagnosis of pathologies with a possible better outcome.

Supplementary Materials: The following are available online at http://www.mdpi.com/2076-3417/10/11/3867/s1, Figure S1: PCR assay to test PDMS compatibility; Figure S2: optimization of silanization time; Figure S3: fluorescent microRNAs adsorption on microdevices.

Author Contributions: Conceptualization, L.L., C.P. (Cristina Potrich), M.C., S.L.M.; methodology, L.L., C.P. (Cristina Potrich), S.L.M.; software, L.L.; validation, L.L. and C.P. (Cristina Potrich); formal analysis, L.L.; investigation, L.L., F.B., G.S., L.V., C.P. (Cristina Potrich)); resources, C.F.P. and C.P. (Cecilia Pederzolli); data curation, L.L. and C.P. (Cristina Potrich); writing-original draft preparation, L.L. and C.P. (Cristina Potrich); writing-review and editing, all authors; visualization, L.L. and C.P. (Cristina Potrich); supervision, L.L., C.P. (Cristina Potrich), M.C., S.L.M., C.F.P., C.P. (Cecilia Pederzolli); project administration, L.L., C.P. (Cristina Potrich), M.C., S.L.M.; funding acquisition, C.F.P. and C.P. (Cecilia Pederzolli). All authors have read and agreed to the published version of the manuscript.

Funding: This research was funded by DEFLeCT ("advanced platform for the early detection of not small cells lung cancer") project, financed by Piedmont Region in the framework of "Health and Well-Being" Platform project.

Acknowledgments: The authors want to acknowledge Andrea Piscitelli and Alessio Testa for their help on micro fabrication process.

Conflicts of Interest: The authors declare no conflict of interest. The funders had no role in the design of the study; in the collection, analyses, or interpretation of data; in the writing of the manuscript, or in the decision to publish the results.

\section{References}

1. Guibert, N.; Pradines, A.; Favre, G.; Mazieres, J. Current and future applications of liquid biopsy in nonsmall cell lung cancer from early to advanced stages. Eur. Respir. Rev. Off. J. Eur. Respir. Soc. 2020, 29, 190052. [CrossRef] [PubMed]

2. Samandari, M.; Julia, M.G.; Rice, A.; Chronopoulos, A.; Hernandez, A.E.D.R. Liquid biopsies for management of pancreatic cancer. Transl. Res. 2018, 201, 98-127. [CrossRef] [PubMed]

3. Yang, Y.; Chen, Y.; Tang, H.; Zong, N.; Jiang, X. Microfluidics for Biomedical Analysis. Small Methods 2019, 4, 1900451. [CrossRef]

4. Solanki, S.; Pandey, C.M.; Gupta, R.K.; Malhotra, B.D. Emerging Trends in Microfluidics Based Devices. Biotechnol. J. 2020, 15, 1900279. [CrossRef] [PubMed]

5. Li, W.; Wang, H.; Zhao, Z.; Gao, H.; Liu, C.; Zhu, L.; Wang, C.; Yang, Y. Emerging Nanotechnologies for Liquid Biopsy: The Detection of Circulating Tumor Cells and Extracellular Vesicles. Adv. Mater. 2019, 31, 1805344. [CrossRef]

6. Vaidyanathan, R.; Soon, R.H.; Zhang, P.; Jiang, K.; Lim, C.T. Cancer diagnosis: From tumor to liquid biopsy and beyond. Lab Chip 2019, 19, 11-34. [CrossRef] 
7. Lin, Z.; Luo, G.; Du, W.; Kong, T.; Liu, C.; Liu, Z. Recent Advances in Microfluidic Platforms Applied in Cancer Metastasis: Circulating Tumor Cells' (CTCs) Isolation and Tumor-On-A-Chip. Small 2020, 16, e1903899. [CrossRef]

8. Xu, X.; Wang, J.; Wu, L.; Guo, J.; Song, Y.; Tian, T.; Wang, W.; Zhu, Z.; Yang, C. Microfluidic Single-Cell Omics Analysis. Small 2019, 16, 1903905. [CrossRef]

9. Wu, Q.; Liu, J.; Wang, X.; Feng, L.; Wu, J.; Zhu, X.; Wen, W.; Gong, X. Organ on a chip: Recent breakthroughs and future prospects. BioMed. Eng. OnLine 2020, 19, 9. [CrossRef]

10. Nguyen, H.V.; Nguyen, V.D.; Nguyen, H.Q.; Chau, T.H.T.; Lee, E.Y.; Seo, T.S. Nucleic acid diagnostics on the total integrated lab-on-a-disc for point-of- care testing. Biosens. Bioelectron. 2019, 141, 111466. [CrossRef]

11. Potrich, C.; Lunelli, L.; Cocuzza, M.; Marasso, S.L.; Pirri, C.; Pederzolli, C. Simple PDMS microdevice for biomedical applications. Talanta 2019, 193, 44-50. [CrossRef] [PubMed]

12. Marasso, S.; Mombello, D.; Cocuzza, M.; Casalena, D.; Ferrante, I.; Nesca, A.; Poiklik, P.; Rekker, K.; Aaspollu, A.; Ferrero, S.; et al. A polymer lab-on-a-chip for genetic analysis using the arrayed primer extension on microarray chips. Biomed. Microdevices 2014, 16, 661-670. [CrossRef] [PubMed]

13. Marasso, S.; Puliafito, A.; Mombello, D.; Benetto, S.; Primo, L.; Bussolino, F.; Pirri, C.; Cocuzza, M. Optimized design and fabrication of a microfluidic platform to study single cells and multicellular aggregates in 3D. Microfluid. Nanofluid. 2017, 21, 29. [CrossRef]

14. Johnston, I.; McCluskey, D.; Tan, C.; Tracey, M. Mechanical characterization of bulk Sylgard 184 for microfluidics and microengineering. J. Micromech. Microeng. 2014, 24, 035017. [CrossRef]

15. Meng, L.; Turner, A.; Mak, W. Soft and flexible material-based affinity sensors. Biotechnol. Adv. 2020, 39, 107398. [CrossRef]

16. Belanger, M.; Marois, Y. Hemocompatibility, Biocompatibility, Inflammatory and in Vivo Studies of Primary Reference Materials Low-Density Polyethylene and Polydimethylsiloxane: A Review. J. Biomed. Mater. Res. 2001, 58, 467-477. [CrossRef]

17. Mata, A.; Fleischman, A.; Roy, S. Characterization of polydimethylsiloxane (PDMS) properties for biomedical micro/nanosystems. Biomed. Microdevices 2005, 7, 281-293. [CrossRef]

18. Pasquardini, L.; Potrich, C.; Quaglio, M.; Lamberti, A.; Guastella, S.; Lunelli, L.; Cocuzza, M.; Vanzetti, L.; Pirri, C.F.; Pederzolli, C. Solid phase DNAextraction on PDMS and direct amplification. Lab Chip 2011, 11, 4029-4035. [CrossRef]

19. Potrich, C.; Vaghi, V.; Lunelli, L.; Pasquardini, L.; Santini, G.; Ottone, C.; Quaglio, M.; Cocuzza, M.; Pirri, C.F.; Ferracin, M.; et al. OncomiR detection in circulating body fluids: A PDMS microdevice perspective. Lab Chip 2014, 14, 4067-4075. [CrossRef]

20. Santini, G.; Potrich, C.; Lunelli, L.; Vanzetti, L.; Marasso, S.; Cocuzza, M.; Pirri, C.; Pederzolli, C. miRNA purification with an optimized PDMS microdevice: Toward the direct purification of low abundant circulating biomarkers. Biophys. Chem. 2017, 229, 142-150. [CrossRef]

21. Makamba, H.; Kim, J.; Lim, K.; Park, N.; Hahn, J. Surface modification of poly(dimethylsiloxane) microchannels. Electrophoresis 2003, 24, 3607-3619. [CrossRef] [PubMed]

22. Król, B.; Pielichowska, K.; Król, P.; Chmielarz, P. Polyurethane cationomers modified by polysiloxane. Polym. Adv. Technol. 2017, 28, 1366-1374. [CrossRef]

23. Schindelin, J.; Arganda-Carreras, I.; Frise, E.; Kaynig, V.; Longair, M.; Pietzsch, T.; Preibisch, S.; Rueden, C.; Saalfeld, S.; Schmid, B.; et al. Fiji: An open-source platform for biological-image analysis. Nat. Methods 2012, 9, 676-682. [CrossRef] [PubMed]

24. Beamson, G.; Briggs, D. High Resolution XPS of Organic Polymers, the Scienta ESCA300 Database; Wiley: Chichester, UK, 1992; p. 295.

25. Speranza, G.; Canteri, R. RxpsG a new open project for Photoelectron and Electron Spectroscopy data processing. Softwarex 2019, 10, 100282. [CrossRef]

26. Tanaka, T.; Sakai, R.; Kobayashi, R.; Hatakeyama, K.; Matsunaga, T. Contributions of phosphate to dna adsorption/desorption behaviors on aminosilane-modified magnetic nanoparticles. Langmuir 2009, 25, 2956-2961. [CrossRef]

27. Lunelli, L.; Caradonna, F.; Potrich, C.; Piotto, C.; Bettotti, P.; Vanzetti, L.; Pederzolli, C.; Guella, G. A new silanizing agent tailored to surface bio-functionalization. Colloids Surf. B Biointerfaces 2019, 181, 166-173. [CrossRef] 
28. Shimomura, A.; Shiino, S.; Kawauchi, J.; Takizawa, S.; Sakamoto, H.; Matsuzaki, J.; Ono, M.; Takeshita, F.; Niida, S.; Shimizu, C.; et al. Novel combination of serum microRNA for detecting breast cancer in the early stage. Cancer Sci. 2016, 107, 326-334. [CrossRef]

29. Bhagirath, D.; Yang, T.; Bucay, N.; Sekhon, K.; Majid, S.; Shahryari, V.; Dahiya, R.; Tanaka, Y.; Saini, S. microRNA-1246 Is an Exosomal Biomarker for Aggressive Prostate Cancer. Cancer Res. 2018, 78, 1833-1844. [CrossRef]

30. Fan, L.; Wang, J.; Cao, Q.; Ding, X.; Li, B. Aberrant miR-1246 expression promotes radioresistance in non-small cell lung cancer: A potential prognostic biomarker and radiotherapy sensitization target. Am. J. Cancer Res. 2020, 10, 314-335.

31. Santini, G.; Potrich, C.; Lunelli, L.; Pasquardini, L.; Vaghi, V.; Pederzolli, C. Innovative microRNA purification based on surface properties modulation. Colloids Surf. B Biointerfaces 2014, 116, 160-168. [CrossRef]

32. Hall, H.K.J. Correlation of the Base Strengths of Amines. J. Am. Chem. Soc. 1957, 79, 5441-5444. [CrossRef]

33. Fears, K.P.; Creager, S.E.; Latour, R.A. Determination of the Surface pK of Carboxylic- and AmineTerminated Alkanethiols Using Surface Plasmon Resonance. Langmuir 2008, 24, 837-843. [CrossRef] [PubMed]

34. Vezenov, D.; Noy, A.; Rozsnyai, L.; Lieber, C. Force Titrations and Ionization State Sensitive Imaging of Functional Groups in Aqueous Solutions by Chemical Force Microscopy. J. Am. Chem. Soc. 1997, 119, 2006-2015. [CrossRef]

35. Vezenov, D.; Noy, A.; Lieber, C. Chemical Force Microscopy: Force Spectroscopy and Imaging of Complex Interactions in Molecular Assemblies. In Handbook of Molecular Force Spectroscopy; Noy, A., Ed.; Springer: Boston, MA, USA, 2007; p. 123.

36. Crabtree, H.J.; Lauzon, J.; Morrissey, Y.C.; Taylor, B.J.; Liang, T.; Johnstone, R.W.; Stickel, A.J.; Manage, D.P.; Atrazhev, A.; Backhouse, C.J.; et al. Inhibition of on-chip PCR using PDMS-glass hybrid microfluidic chips. Microfluid. Nanofluid. 2012, 13, 383-398. [CrossRef]

37. Potrich, C.; Lunelli, L.; Forti, S.; Vozzi, D.; Pasquardini, L.; Vanzetti, L.; Panciatichi, C.; Anderle, M.; Pederzolli, C. Effect of materials for micro-electro-mechanical systems on PCR yield. Eur. Biophys. J. 2010, 39, 979-986. [CrossRef] [PubMed]

38. Louette, P.; Bodino, F.; Pireaux, J.-J. Poly (dimethyl siloxane) (PDMS) XPS Reference Core Level and Energy Loss Spectra. Surf. Sci. Spectra 2005, 12, 38-43. [CrossRef]

39. Chen, X.; Hu, Z.; Wang, W.; Ba, Y.; Ma, L.; Zhang, C.; Wang, C.; Ren, Z.; Zhao, Y.; Wu, S.; et al. Identification of ten serum microRNAs from a genome-wide serum microRNA expression profile as novel noninvasive biomarkers for nonsmall cell lung cancer diagnosis. Int. J. Cancer 2012, 130, 1620-1628. [CrossRef]

40. Cui, M.; Wang, H.; Yao, X.; Zhang, D.; Xie, Y.; Cui, R.; Zhang, X. Circulating MicroRNAs in Cancer: Potential and Challenge. Front. Genet. 2019, 10, 626. [CrossRef] 